\title{
Fuerzas competitivas que moldean la estrategia en la gerencia del sector MiPyME del distrito de Santa Marta -Magdalena, Colombia-
}

Zoraima Aurelia Donawa Torres Instituto Universitario de Tecnología de Cabimas zdonawa62@hotmail.com

Eugenia Cristina Morales Martínez $z^{2}$ Universidad de Magdalena eugeniam828@gmail.com

DOI: https://doi.org/10.21158/01208160.n84.2018.1919

Fecha de recepción: 18 de septiembre de 2017

Fecha de aprobación: 06 de febrero de 2018

Cómo citar este artículo / To reference this article / Comment citer cet article / Para citar este artigo:

Donawa Torres, Z. A. y Morales Martínez, E. C. (2018). Fuerzas competitivas que moldean la estrategia en la gerencia del sector mipyme del distrito de Santa Marta -Magdalena, Colombia-. Revista EAN, 84, (pp. 97-108). DOI: https://doi. org/10.21158/01208160.n84.2018.1919

\section{- Resumen}

Las estrategias gerenciales promueven la productividad, impulsando a las empresas a satisfacer las necesidades del entorno, incrementando así su productividad. Teniendo esto en cuenta, el objetivo de esta investigación se centra en identificar la aplicación de tácticas ante las fuerzas competitivas que moldean la estrategia en la gerencia del sector de las MiPYMEs del distrito de Santa Marta -Colombia-. Como base teórica se utilizaron los planteamientos de autores como Fred David (2013), Michael Porter (2009), Richard Daft (2011) y Charles Hill y Gareth Jones (2009), entre otros y en el aspecto metodológico se trabajó desde el parámetro positivista, no experimental, transeccional descriptivo y de campo. La población objeto del estudio estuvo constituida por 40 empleados de cinco empresas y los datos fueron recolectados a través de un cuestionario tipo Likert. Los resultados indicaron que los gerentes de las empresas aplican estrategias para enfrentar las fuerzas competitivas y fortalecer el éxito en el mercado manteniendo a largo plazo su posición competitiva a costa de sus rivales en el sector.

\section{Palabras clave}

Estrategias, gerencia, competitividad, fuerzas competitivas.

\footnotetext{
${ }^{1}$ Politóloga, Maestría en Gerencia de empresas, Doctorado en Ciencias Gerenciales, Post Doctorado en Gerencia de las Organizaciones. Docente Agregado e Investigadora del Instituto Universitario de Tecnología de Cabimas, Venezuela. ORCID: http://orcid.org/0000-0002-6142-9500

${ }^{2}$ Doctora en Gestión de la Innovación. Docente catedrático e Investigadora de la Universidad de Magdalena, Colombia.
} 


\title{
Competitive forces which shape the management strategy at the MIPyME sector in Santa Marta-Magdalena, Colombia-
}

\begin{abstract}
Management strategies promote productivity, leading companies to satisfy their environment needs and increasing productivity rates. Based on this, this study aims at identifying the application of different tactics to existing competitive forces which shape the management strategy at the mipyme sector in Santa Marta, Colombia. As theoretical bases, different authors' opinions were used such as Fred David (2013), Michael Porter (2009), Richard Daft (2011) and Charles Hill and Gareth Jones (2009), among others, and the positivist parameter applied as a non-experiential, transectional descriptive and field methodological aspect. The population sample used as object of study involved 40 employees from 5 companies and data were collected from the use of Likert questionnaire. The outcomes showed that company managers applied strategies to face competitive forces and to strengthen market success, maintaining their competitive market positioning above their rivals from the same industrial sector.
\end{abstract}

Key words: Sstrategies, management, competitiveness, competitive forces

\section{Les forces concurrentielles façonnant les stratégies de gestion du secteur des microentreprises mipyme du Santa Marta-Magdalena, Colombia-}

Résumé.Les stratégies de gestion favorisent la productivité en encourageant les entreprises à répondre aux besoins de leur environnement, augmentant de fait leur productivité. L'objectif de cette recherche est d'identifier la mise en place de tactiques pouvant faire face aux forces compétitives façonnant les stratégies de gestion du secteur des microentreprises du district de Santa Marta - Colombie. Les bases théoriques utilisées référencent des auteurs comme Fred David (2013), Michael Porter (2009), Richard Daft (2011) et Charles Hill et Gareth Jones (2009), entre autres. L'aspect méthodologique retenu est de type positiviste, non expérimental, transectionnel descriptif. La population étudiée est composée de 40 employés de cinq entreprises différentes et les données ont été recueillies au moyen d'un questionnaire Likert. Les résultats indiquent que les dirigeants de ces entreprises appliquent des stratégies pour faire face à la concurrence et renforcer leur succès sur le marché en maintenant leur position sur le long terme au détriment de leurs concurrents.

Mots clefs: Stratégies, gestion, compétitivité, forces compétitives.

\section{Forças competitivas que moldam a estratégia na gerência do setor mipyme do Santa Marta-Magdalena, Colômbia-}

Resumo. As estratégias gerenciais promovem a produtividade, impulsionando as empresas a satisfazer as necessidades do meio, incrementando assim sua produtividade. Considerando isto, o objetivo desta pesquisa se centra em identificar o aplicativo de tácticas ante as forças competitivas que moldam a estratégia na gerência do setor das MiPYMEs do distrito de Santa Marta, Colômbia. Como base teórica se utilizaram as propostas de autores como Fred David (2013), Michael Porter (2009), Richard Daft (2011) e Charles Hill e Gareth Jones (2009), entre outros e no aspecto metodológico se trabalhou desde o parâmetro positivista, não experimental, transeccional descritivo e de campo. A população objeto de estudo esteve constituída por 40 empregados de cinco empresas e os dados foram coletados através de um questionário tipo Likert. Os resultados indicam que os gerentes das empresas aplicam estratégias para enfrentar as forças competitivas e fortalecer o sucesso no mercado mantendo em longo prazo sua posição competitiva a custa de seus rivais no setor.

Palavras-chave: Estratégias, gerência, competitividade, forças competitivas. 


\section{Introducción}

$E_{8}^{1}$ fenómeno económico conocido como globalización está impulsando a la gerencia de las organizaciones hacia una gestión de la competitividad, independiente del tamaño y ubicación de la empresa, lo cual ha originado la inminente preocupación por investigar y precisar estrategias competitivas para el crecimiento económico y desarrollo social de un país en el que no están exentas las micro, pequeñas y medianas empresas -MiPYMEs-.

A este respecto, Reta (2008) define la competitividad como la capacidad de cualquier empresa, pública o privada, para perseverar en forma ordenada y precisa ventajas comparativas que le permitan alcanzar, mantener y desarrollar determinada posición en el entorno social y económico. No obstante, dentro de este contexto, están inmersas las MiPYMEs como unidades de explotación dentro del engranaje socioeconómico de Colombia, en variables como número de establecimientos, empleo, producción, distribución del ingreso, entre otros indicadores, las cuales realizan actividades empresariales, agropecuarias, industriales, comerciales o de servicios, rurales o urbanas. Las MiPYMEs en Colombia forman parte de la estructura empresarial contribuyendo en gran manera a la generación de empleo y ocupación de la mano de obra en el país.
Teniendo en cuenta estas acotaciones, resulta inminente admitir la gran importancia de la competitividad de las MiPYMEs reflejada en la productividad, en la que una nación, región o sector usa sus recursos naturales y humanos para crear y mantener un buen nivel de vida para sus habitantes. En este sentido, la gerencia en estas empresas debe tomar en consideración estrategias competitivas relacionadas con su medio ambiente para hacerles frente a los desafíos del entorno y llevar a cabo la coordinación de los recursos en función de los objetivos a fin de utilizar estrategias con criterios adecuados para el desarrollo integral de la empresa.

Las fuerzas competitivas de Porter (2009) permiten a la gerencia de las empresas enfrentar con estrategias los factores externos que pueden afectar la posición de éxito a largo plazo dentro de la industria y de superar a los competidores.

Sobre la base de estas consideraciones, el objetivo de esta investigación se centra en identificar la aplicación de estrategias ante las fuerzas competitivas que moldean la estrategia en la gerencia del sector de las MiPYMEs del distrito de Santa Marta. Para ello, se elabora un enfoque teórico de algunos autores sobre el tema, se elabora un marco metodológico para la recolección de datos de datos en cinco empresas pertenecientes al sector, se realiza un análisis e interpretación de los resultados y finalmente, se establecen las conclusiones de esta investigación. 


\section{Competitividad}

T a competitividad regional se refleja en La capacidad de una economía regional para atraer y mantener la localización de empresas, con cuotas de participación sectorial estables y crecientes, lo cual posibilita el mantenimiento de unos estándares de calidad y nivel de vida para todos sus habitantes (Saracho, 2005). Las empresas compiten para ganarse la preferencia de los consumidores, proveedores y clientes. En tal sentido, para ser competitivas, necesitan tener un desempeño mayor que otras, pues se disputan los mismos clientes. Una organización es competitiva cuando tiene alguna ventaja sobre sus competidores.

Esta ventaja competitiva, señala Amaru (2009), hace que una empresa sea preferida por los clientes, que sea más competente en alguna forma de relación con el ambiente o que disponga de algún recurso singular. La ventaja competitiva hace que una empresa sea preferida por los clientes, que sea más competente en alguna forma de relación con el ambiente o que disponga de algún recurso singular.

En una empresa, la ventaja competitiva proviene de la capacidad para satisfacer las necesidades de los consumidores con más eficacia, con productos o servicios que los consumidores aprecien mucho o con más eficiencia, a un menor costo. Satisfacer con mayor eficacia las necesidades de los consumidores se puede traducir en la capacidad de pedir un precio más elevado (Thompson, Peteraf, Gamble y Strickland, 2012). Por su parte, Dessler (2009) explica que una ventaja competitiva se define como todos los factores que permiten que una organización distinga su producto o servicio de los de la competencia, con el objetivo de aumentar su participación en el mercado.

\section{Fuerzas competitivas que moldean la estrategia de las empresas}

T as cinco fuerzas que moldean la estraLegia de las empresas (Porter, 2009) han sido analizadas por varios autores. Koontz, Weihrich y Cannice (2012) explican que Porter sugiere que la formulación de estrategias requiere un análisis del atractivo de una industria y la posición de la compañía dentro de ella, y este análisis se convierte en la base para formular las estrategias genéricas. Sobre la base del análisis de la industria, una compañía puede adoptar estrategias genéricas, que se denominan así porque pueden adecuarse a un amplio nivel para diferentes tipos de organizaciones; sin embargo, cualquier empresa puede usar más de una estrategia.

Hill y Jones (2009) consideran que el modelo de Porter ayuda a los administradores a realizar este análisis para identificar las oportunidades y amenazas. Por otro lado, Daft (2011) señala que es un modelo eficaz para formular la estrategia y permite determinar la posición de una empresa frente a los competidores. Explica que Porter estudió varias organizaciones de negocios y propuso que los gerentes pueden formular una estrategia que permita a 
la organización tener una mayor rentabilidad y ser menos vulnerable si entienden las cinco fuerzas del entorno de la industria.

Por su parte, Porter (2009) expresa que la labor fundamental del gerente o estratega es hacer frente a la competitividad, y conseguir y mantener una ventaja competitiva sobre el resto de empresas es probablemente el objetivo directo o indirecto de la dirección de cualquier empresa. Enuncia que la competencia por obtener beneficios va más allá de los competidores directos o rivales consolidados de una empresa. El autor arguye que la ampliación de la rivalidad que se origina de la combinación de cinco fuerzas define la estructura de una empresa y moldea la naturaleza de la interacción competitiva dentro de ella. Así mismo, advierte que la estructura de una empresa, manifestada en las fuerzas competitivas, marca su rentabilidad a medio y a largo plazo.

\subsection{Amenaza de nuevos aspirantes}

Porter (2009) explica que los nuevos aspirantes a entrar en una empresa aportan una nueva capacidad y un deseo de obtener una cuota de mercado que ejerce presión en los precios, los costes y el índice de inversión necesario para competir. Advierte que cuando los nuevos aspirantes proceden de otras empresas y deciden diversificarse, pueden influir en la capacidad existente y en los flujos de liquidez para estimular la competitividad.

En palabras de David (2013), las barreras contra el ingreso de nuevos aspirantes pueden incluir la necesidad de lograr rápidamente economías de escala, la necesidad de obtener tecnología y conocimientos técnicos especializados, la falta de experiencia, una fuerte lealtad por parte de los consumidores, sólidas preferencias por determinadas marcas, grandes requerimientos de capital, carencia de canales de distribución adecuados, políticas de regulación gubernamental, aranceles, dificultad de acceso a las materias primas, posesión de patentes, ubicaciones poco deseables, el contraataque por parte de empresas bien afianzadas y la potencial saturación del mercado.

Hill y Jones (2009) indican que los competidores son organizaciones que producen bienes y servicios parecidos a los bienes y servicios de una organización determinada. En otras palabras, los competidores son organizaciones que compiten por los mismos clientes. Los competidores potenciales son organizaciones que por el momento no están dentro del entorno específico de un sector, pero podrían entrar en él si lo quisieran.

No obstante, explica David (2003), a pesar de las diversas barreras de ingreso, algunas empresas nuevas entran en las industrias con productos de excelente calidad, precios bajos y recursos de mercadotecnia importantes; por tanto, el trabajo del estratega consiste en identificar las empresas nuevas que tienen la posibilidad de ingresar al mercado, vigilar las estrategias de las nuevas empresas rivales, contraatacar si es necesario y obtener provecho de las fortalezas y oportunidades existentes.

El trabajo de la gerencia consiste en identificar las empresas nuevas, que tienen la posibilidad de ingresar al mercado, vigilar las estrategias de las nuevas empresas rivales, contraatacar si es necesario y obtener provecho de las fortalezas y oportunidades existentes. De esta manera, Porter (2009) hace hincapié en que el reto es encontrar la forma de superar las barreras de

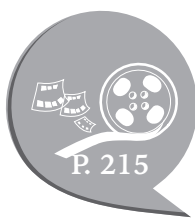


entrada sin anular, con grandes inversiones, la rentabilidad de participar en el mercado. Así mismo, advierte que, cuando la amenaza es real, los gerentes deben reducir los precios o incrementar la inversión para detener a los nuevos competidores.

Es importante acotar que Hitt, Ireland y Hoskisson (2006) definen la estrategia de liderazgo en costos como un conjunto integrado de acciones que desempeña la empresa para producir bienes o servicios que tengan características aceptables para los clientes, al costo más bajo posible, en relación con el de sus competidores. Esta estrategia sirve como una barrera importante contra la entrada de posibles competidores. Los nuevos entrantes deben tener la capacidad y la disposición para aceptar rendimientos que no rebasen el promedio mientras adquieren la experiencia necesaria para acercarse a la eficiencia de la líder en costos.

\subsection{La influencia de los proveedores}

Revela Porter (2009) que los proveedores acaparan más valor para sí mismos al cobrar un precio elevado, limitar la calidad o los servicios o al traspasar sus costes a los participantes de la empresa. Los proveedores poderosos, incluidos los que proporcionan mano de obra, pueden sacar la máxima rentabilidad de una industria que no pueda traspasar un incremento del coste al precio final. El poder de negociación de los proveedores, las organizaciones que proporcionan insumos a la industria como materiales, servicios y mano de obra, se refiere a la capacidad de estos para aumentar los precios de los insumos o elevar de otro modo los costos de la industria, por ejemplo, al ofrecer insumos de baja calidad o un servicio deficiente (Hill y Jones, 2009).
Del mismo modo, David (2013) considera que el poder de negociación de los proveedores también afecta la intensidad de la competencia en una industria y que las compañías pueden buscar una estrategia de integración hacia atrás para obtener el control o apropiarse de los proveedores. Esta estrategia es especialmente efectiva cuando los proveedores no son confiables, muy costosos o incapaces de satisfacer las necesidades de la empresa de manera constante. Por lo general, las compañías pueden negociar términos más favorables con los proveedores si la integración hacia atrás es una estrategia comúnmente usada entre empresas rivales de una industria.

Hitt, Ireland y Hoskisson (2006) acotan que el líder en costos opera con márgenes más grandes que los de sus competidores. Entre otros beneficios, los grandes márgenes del líder en costos, con relación a los de los competidores, le permiten absorber los incrementos de precios de sus proveedores. Cuando el costo de los suministros de una industria registra incrementos sustantivos, solo el líder en costos estará en posición de pagar precios más altos y de seguir obteniendo rendimientos promedio o superiores a este. Por otra parte, si un líder en costos es poderoso, podría obligar a sus proveedores a mantener bajos sus precios, con lo cual disminuirían los márgenes de los proveedores.

\subsection{La influencia de los compradores}

Señala Porter (2009) que los clientes influyentes, es decir, la otra cara de los proveedores influyentes, pueden acaparar más valor al forzar los precios a la baja, exigiendo mejor calidad o más prestaciones, lo cual hace subir los costes, y enfrentando, en general, a los distintos participantes de una empresa. Los compradores son poderosos 
si cuentan con influencia negociadora sobre los participantes de una empresa, especialmente si son sensibles a los precios, porque emplean ese peso para ejercer presión en la reducción de precios.

En palabras de Hill y Jones (2009), los compradores de una empresa pueden ser los clientes individuales que consumen sus productos en última instancia -los usuarios finales) o las compañías que distribuyen los productos de una industria a los usuarios finales como comercializadores al menudeo y mayoreo. Cuando los clientes están concentrados, son muchos o compran por volumen, su poder de negociación representa una importante fuerza que afecta la intensidad de la competencia en una industria. Si el poder de negociación de los consumidores es fuerte, las compañías rivales podrían tratar de responder ofreciendo garantías extendidas o servicios especiales para conseguir su lealtad (David, 2013).

El poder de negociación de los consumidores es también mayor, según David (2003), cuando los productos que se adquieren son estándar o poco diferenciados. Cuando esto ocurre, los consumidores negocian precio de venta, cobertura de la garantía y paquetes adicionales en mayor grado.

Como estrategia, Hitt, Ireland y Hoskisson (2006) explican que el carácter exclusivo de los bienes y servicios diferenciados disminuye la sensibilidad de los clientes a los incrementos de precio, quienes estarán dispuestos a aceptar un aumento de este mientras un producto siga satisfaciendo aquellas de sus necesidades que perciben como únicas mejor que los que ofrecen los competidores.

\subsection{La amenaza de los sustitutivos}

Porter (2009) distingue que un sustitutivo realiza una función idéntica o similar a la del producto de una empresa, pero de distinta manera. Explica que se tiene a un sustitutivo cuando se puede prescindir de ese producto, se prefiere comprar uno usado en vez de adquirir otro nuevo o lo fabrica el consumidor -trae el producto o servicio a la casa-. Considera que cuando la amenaza de sustitutivos es elevada, la rentabilidad de la empresa se reciente, pues los productos sustitutivos no solo limitan los beneficios en tiempos normales, sino que también reducen la bonanza que una empresa puede capitalizar en los buenos tiempos.

La amenaza de los productos sustitutos, señalan Hill y Jones (2009), se refleja en los productos de diferentes negocios o industrias que pueden satisfacer necesidades semejantes de los clientes. La existencia de sustitutos cercanos es una amenaza competitiva poderosa, porque limita el precio que pueden cobrar las compañías de una industria por su producto $\mathrm{y}$, por consiguiente, la rentabilidad de la industria.

En palabras de David (2013), las presiones competitivas que surgen por los productos sustitutos aumentan a medida que el precio relativo de estos disminuye, por lo cual el costo en que incurren los consumidores por cambiar a ellos también se reduce. La fuerza competitiva de los productos sustitutos se mide mejor por la participación de mercado que logran esos productos y por los planes que hacen las empresas fabricantes para incrementar su capacidad y su penetración de mercado.

Los estrategas deberían prestar especial atención a los cambios en otras industrias, que los convierten en sustitutivos atractivos. Así mismo, la fortaleza competitiva de los productos sustitutos se mide mejor por los 
avances que estos obtienen en la participación en el mercado y por los planes que tienen las empresas para aumentar su capacidad y penetración en el mercado (David, 2003).

Hitt, Ireland y Hoskisson (2006) enuncian que las empresas que venden bienes y servicios de marca a los clientes leales están posicionadas de forma efectiva contra los productos sustitutos. Por otra parte, las empresas cuyos clientes no son leales a su marca tienen más probabilidades de que estos decidan cambiar sus productos por otros que ofrecen atributos diferenciados que cumplen con la misma función -sobre todo si el sustituto tiene un precio más bajoo por productos que ofrecen más atributos y desempeñan funciones más atractivas.

\subsection{Rivalidad entre competidores existentes}

La rivalidad entre los competidores existentes, a juicio de Porter (2009), adopta muchas formas conocidas, entre las cuales se incluyen los descuentos en los precios, nuevas mejoras en el producto, campañas de publicidad y mejoras en el servicio. Una rivalidad elevada limita el rendimiento de una empresa. El grado de rivalidad que hace descender el potencial de beneficio de una industria depende, primero, de la intensidad con la que las empresas compiten, y segundo, de la base sobre la que están compitiendo. La intensidad de la rivalidad es mayor si los competidores son numerosos o muy parecidos en tamaño e influencia: en estos casos, los rivales tienen dificultades para evitar interferir en sus asuntos (Porter, 2009).

Dado que las empresas de una industria dependen unas de otras, las acciones que emprende una suelen despertar respuestas de otros competidores. En muchas industrias, las empresas compiten activamente entre sí. La rivalidad competitiva se intensifica cuando las acciones de un competidor son un reto para otra empresa o cuando la empresa reconoce una oportunidad para mejorar su posición de mercado (Hitt, Ireland y Hoskisson, 2006). Los clientes suelen ser compradores leales de productos que se diferencian en formas que tienen significado para ellos. A medida que los clientes se vuelvan más leales a una marca, su sensibilidad a los aumentos de precios irá disminuyendo. La relación entre la lealtad a la marca y la sensibilidad al precio aísla a la empresa de los rivales que compiten contra ella.

La rivalidad entre empresas competidoras suele ser la más poderosa de las cinco fuerzas competitivas (David, 2013). Las estrategias de una empresa solo pueden tener éxito en la medida en que le proporcionen una ventaja competitiva sobre las estrategias de las empresas rivales. Los cambios de estrategia que ponga en práctica una empresa podrían dar lugar a represalias como la reducción de precios, las mejoras a la calidad, la introducción de nuevas características en los productos, el ofrecimiento de servicios, la extensión de garantías y el aumento de publicidad.

La lucha competitiva se puede basar en precios, diseño del producto, gastos de publicidad y promoción, esfuerzos de ventas directas, y servicio y apoyo después de las ventas. Una rivalidad más intensa implica precios más bajos y mayor gasto en armas competitivas no relacionadas con el precio o ambas. Como la rivalidad intensa reduce los precios e incrementa los costos, recorta las ganancias de una industria. Por tanto, la rivalidad intensa entre compañías establecidas constituye una poderosa amenaza para la rentabilidad (Hill y Jones, 2009). 


\section{Metodología}

T a investigación se realizó bajo el enfoque epistemológico positivista, el tipo de investigación fue descriptivo, el diseño transeccional o transversal y de campo y se recurrió a los métodos de análisis y síntesis. La población de tipo finita estuvo compuesta por cuarenta empleados ordinarios, de ambos sexos, de diferentes edades, quienes ocupaban cargos de gerente y personal administrativo de las MiPYMEs del distrito de Santa Marta (Tabla 1).
Para la recolección de datos, se elaboró un cuestionario validado por expertos, con una confiabilidad arrojada por el coeficiente alfa Cronbach de 0.97. El cuestionario estaba constituido por quince ítems con categoría de respuesta tipo Likert. Los datos se agruparon en tablas de frecuencia para aplicar el método de investigación de análisis y síntesis.

Tabla 1. Población

\begin{tabular}{|l|c|}
\hline \multicolumn{1}{|c|}{ Empresa } & Población \\
\hline Unidad de Atención en Pacientes en Estado Crítico & 5 \\
\hline Refrielectric S. A. & 3 \\
\hline Inversiones Agrícolas y Comerciales & 8 \\
\hline Tecnoaguas & 4 \\
\hline Morano Grupo S. A. S. & 10 \\
\hline Total & 40 \\
\hline
\end{tabular}

Fuente. Elaboración propia.

\section{Resultados}

1 identificar la aplicación de las fuerzas
en la gerencia del sector de las MiPyMEs del
distrito de Santa Marta, se puede evidenciar
que 12 de los empleados que representan el
$30 \%$ de los encuestados y 11 de los empleados
que representan el $28 \%$ de los encuestados
seleccionaron las categorías de respuestas
positivas al escoger las afirmaciones sobre la

aplicación de estrategias contra la amenaza de nuevos aspirantes (Tabla 2). Del mismo modo, se refleja que 15 de los empleados que representan el $38 \%$ de los empleados encuestados y 13 de los empleados que representan el $33 \%$ de los encuestados consideran que la gerencia aplica estrategias de integración ante la influencia de los proveedores.

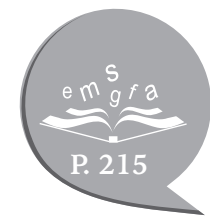


Tabla 2. Fuerzas competitivas que moldean la estrategia

\begin{tabular}{|c|c|c|c|c|c|c|c|c|c|c|c|c|}
\hline \multirow{2}{*}{$\begin{array}{l}\text { Categoría de } \\
\text { respuesta }\end{array}$} & \multicolumn{2}{|c|}{ Aspirantes } & \multicolumn{2}{|c|}{ Proveedor } & \multicolumn{2}{|c|}{ Comprador } & \multicolumn{2}{|c|}{ Sustitutivos } & \multicolumn{2}{|c|}{ Rivalidad } & \multirow{2}{*}{$\square$} & \multirow{2}{*}{$\%$} \\
\hline & $\mathrm{Fi}$ & $\%$ & $\mathrm{Fi}$ & $\%$ & $\mathrm{Fi}$ & $\%$ & $\mathrm{Fi}$ & $\%$ & $\mathrm{Fi}$ & $\%$ & & \\
\hline $\begin{array}{l}\text { Completamente } \\
\text { verdadero }\end{array}$ & 11 & 28 & 13 & 33 & 14 & 35 & 12 & 30 & 13 & 33 & 13 & 32 \\
\hline Verdadero & 12 & 30 & 15 & 38 & 12 & 30 & 19 & 48 & 14 & 35 & 14 & 36 \\
\hline $\begin{array}{l}\text { Ni falso ni } \\
\text { verdadero }\end{array}$ & 2 & 5 & 3 & 8 & 2 & 5 & 2 & 5 & 2 & 5 & 2 & 6 \\
\hline Falso & 9 & 23 & 4 & 10 & 6 & 15 & 4 & 10 & 7 & 18 & 6 & 15 \\
\hline $\begin{array}{l}\text { Completamente } \\
\text { falso }\end{array}$ & 6 & 15 & 5 & 13 & 6 & 15 & 3 & 8 & 4 & 10 & 5 & 12 \\
\hline Total & 40 & 100 & 40 & 100 & 40 & 100 & 40 & 100 & 40 & 100 & 40 & 100 \\
\hline
\end{tabular}

Fuente. Elaboración propia.

En el mismo orden de ideas, y de acuerdo con los resultados logrados, 14 de los empleados que representan el $35 \%$ de los encuestados y 12 de los empleados que representan el $30 \%$ de los encuestados declaran que la gerencia posee planes estratégicos para la influencia de los compradores. Así mismo, cabe destacar que 19 de los empleados que representan el $48 \%$ de los empleados y 12 de los empleados que representan el $30 \%$ perciben que la gerencia presta atención ante la amenaza de los sustitutivos. Y finalmente, se puede acotar que 14 de los empleados que representan el $35 \%$ de los encuestados y 13 de los empleados que representan el $33 \%$ consideran que la gerencia emprende acciones para hacerle frente a la rivalidad entre competidores existentes.

Finalmente, se debe acotar que los resultados reflejados anteriormente ponen en evidencia que de los 40 empleados, 27 que representan el $68 \%$ de los encuestados consideran que la gerencia de las empresas estudiadas aplica estrategias para el desarrollo de su competitividad (Figura 1).

Figura 1. Fuerzas competitivas que moldean la estrategia

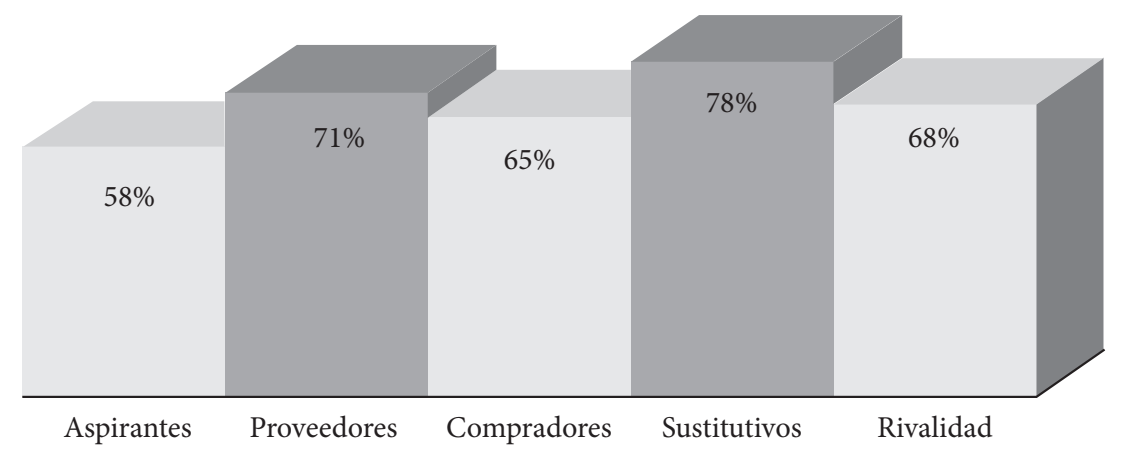

Fuente. Elaboración propia. 
La evidencia presentada anteriormente permite inferir que al sumar las categorías de respuestas positivas, 23 de los empleados que representan el $58 \%$ de los encuestados identifican la aplicación de estrategias ante la amenaza de nuevos aspirantes en la gerencia del sector de las MiPYMEs del distrito de Santa Marta. Los empleados perciben que la gerencia se interesa por vigilar las empresas con posibilidades de ingresar al mercado que puedan convertirse en una amenaza, de tal manera que consideren como estrategia disminuir los precios o incrementar la inversión para combatir la posibilidad de que nuevas empresas entren fácilmente y que la intensidad de la competitividad aumente. Así mismo, se preocupa por obtener tecnología y conocimientos técnicos y una fuerte lealtad por parte de los consumidores.

Del mismo modo, cabe notar que 28 de los empleados que representan el $71 \%$ de los encuestados enuncian que la gerencia hace frente a la influencia de los proveedores, pues consideran la negociación de la gerencia con los proveedores para controlarlos utilizando la estrategia de integración hacia atrás, y trata de ser el líder de costos obligando a sus proveedores a mantener bajos los precios. En este sentido, se evidencia que la gerencia disfruta de la lealtad de los proveedores y productores ayudándose entre sí con precios razonables, mejor calidad, desarrollando nuevos servicios, haciendo entregas a tiempo y reduciendo los costos de inventario; de esta manera, mejoran la rentabilidad de todos los interesados a largo plazo.
En el mismo orden de ideas, cabe destacar que 26 de los empleados que representan el $65 \%$ de los encuestados perciben en la gerencia la aplicación de estrategias ante la influencia de los compradores, ofreciendo servicios especiales, logrando la lealtad de los consumidores, negociando el precio de venta, cobertura de la garantía y paquetes adicionales en mayor grado e impulsando el carácter exclusivo de los bienes y servicios. Así mismo, se debe acotar que 31 de los empleados que representan el $78 \%$ de los encuestados consideran que la gerencia aplica estrategias ante la amenaza de sustitutivos, tratando de desarrollar sus productos o servicios para que sean insustituibles con un perfil tal que los hagan más deseables para expandir la capacidad de producción, sus estimados de crecimiento de ventas y utilidades.

Finalmente, se debe expresar que 27 de los empleados que representan el $68 \%$ de los encuestados afirman la aplicación de estrategias gerenciales ante la rivalidad entre compradores existentes, gestionando estratégicamente como la lucha competitiva con los descuentos en los precios para ser más competitivo, impulsando descuentos en los precios de los productos o servicios para competir, mejorando su calidad en la introducción de nuevas características e impulsando campañas de publicidad. 


\section{Conclusiones}

\begin{abstract}
A 1 identificar la aplicación de estraAtegias ante las fuerzas competitivas que moldean la estrategia en la gerencia del sector de las MiPyMEs del distrito de Santa Marta, los resultados permiten inferir que la gerencia de estas empresas aplica estrategias para combatir las fuerzas competitivas, vigila las empresas nuevas que tienen la posibilidad de ingresar al mercado, consideran al proveedor como un colaborador, ofrecen garantías prolongadas o servicios especiales para ganar la lealtad de los clientes, prestan especial atención a los cambios en otras industrias que los convierten en sustitutivos atractivos y están conscientes de que la intensidad de la rivalidad es
\end{abstract}

\section{Referencias}

Amaru, A. C. (2009). Fundamentos de administración: teoría general y proceso administrativo. México: Pearson.

Daft, R. L. (2011). Teoría y diseño organizacional (10. ${ }^{\text {a }}$ ed.). México: Cengage.

David, F. (2003). Administración estratégica. México: Pearson.

David, F. (2013). Conceptos de administración estratégica (14. ${ }^{\mathrm{a}}$ ed.). México: Pearson.

Dessler, G. (2009). Administración de recursos humanos

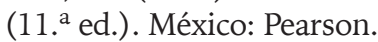

Hill, C. y Jones, G. (2009). Administración estratégica (8. ${ }^{a}$ ed.). México: McGraw- Hill.

Hitt, M., Ireland, D. y Hoskisson, R. (2006). Administración estratégica: competitividad y conceptos de globalización (7. $\left.{ }^{\mathrm{a}} \mathrm{ed}.\right)$. México: Thomsom. mayor si los competidores son numerosos o muy parecidos en tamaño e influencia. Los gerentes de las empresas piensan estratégicamente utilizando la creatividad y la intuición, tomando decisiones para obtener una ventaja competitiva y formulando estrategias frente a las amenazas del mercado.

Se concluye que los gerentes de estas organizaciones accionan para transformar situaciones, coordinando la utilización de los recursos en función de los criterios estratégicos adecuados con el fin de crear una buena posición dentro de la industria, como la intención de mantener y fortalecer el éxito en el mercado manteniendo a largo plazo su posición competitiva a costa de sus rivales en el sector.

Koontz, H., Weihrich, H. y Cannice, M. (2012). Administración: una perspectiva global y empresarial (14. ${ }^{\text {a }}$ ed.). México: McGraw-Hill.

Porter, M. (2009). Ser competitivo. Barcelona: Deusto.

Reta, M. (2008). Políticas para la competitividad. Pyme Hoy, 4, 31-59.

Saracho, J. M. (2005). Un modelo general de gestión por competencias. Santiago de Chile: Ril editores.

Serna, H. (2006). Gerencia estratégica. Bogotá: Panamericana.

Thompson, A., Peteraf, M., Gamble, J. y Strickland, J. (2012). Administración estratégica (18. a ed.). México: McGraw-Hill. 